\title{
Comparison of the defect detection capabilities of flash thermography and vibration excitation shearography
}

By S. G. Pickering* and D.P. Almond*

* Dept. of Mechanical Engineering, University of Bath, Bath, BA2 7AY, UK, S.G.Pickering@bath.ac.uk

\begin{abstract}
The defect detection capabilities of transient thermography and shearography have been compared using optimum excitation methods for each technique: short pulse heating for thermography and vibration excitation using a piezoelectric transducer for shearography. A signal-to-noise ratio and limit of detection analysis has been performed on defect images obtained by the two techniques using the different excitation methods. Test samples considered in this paper are flat-plate samples made from aluminium, mild steel, stainless steel, CFRP and thermoplastic, containing flat-bottomed hole artificial defects of $20 \mathrm{~mm}$ diameter at depths ranging from $0.5 \mathrm{~mm}$ to $3.0 \mathrm{~mm}$.
\end{abstract}

Keywords: infrared thermography, shearography, non-destructive testing

\section{Introduction}

Both flash thermography $[1,2,3]$ and shearography $[4,5,6]$ are non-contactive wide-area optical measurement techniques that are suited to the detection of planar defects parallel to the surface of a sample, e.g. disbonds and delaminations. The purpose of this paper is to provide a comparison of the performances of flash thermography and vibration excitation shearography in testing a variety of sample materials containing flatbottomed back-drilled hole artificial defects.

The technique of flash thermography involves using a short duration ( $1 \mathrm{~ms})$ high intensity flash of light to heat the surface of a test piece. The test piece surface temperature is recorded by an infrared camera and computer system as it decays due to heat being conducted into the part after its deposition on the surface. Subsurface defects reduce the conduction of heat away from the surface and therefore reduce the surface cooling rate compared to that occurring over non-defective regions. Consequently, a surface temperature contrast develops over a defect that can be used to detect a defective region.

Shearography, otherwise known as shearing speckle interferometry, is a technique that uses interferometry to measure the out of plane displacement gradient of a sample's surface. The presence of defects will alter the way in which a sample reacts to an applied stress (in this case created by vibrating the test sample) and this change can be observed using shearography thereby inferring the presence of the defect. In shearography a speckle pattern is applied to the surface of the test sample and the sample is illuminated using expanded collimated (i.e. laser) light. The light is reflected diffusely from the surface of the sample and passes through a lens and shearing device to be recorded by a video camera and processed. The shearing device acts to slightly change the path of half of the rays of light reflected by the speckles, causing the reflected light from neighbouring speckles to overlap and produce an image sheared in the shearing direction.

A reference sheared image is stored with the sample in an unstressed condition and the sample is then stressed. The stressing can take many forms, mechanical strain (e.g. with a vacuum hood or by applying a bending load with a clamp), thermal stress (generated by heating or cooling the sample), or vibration excitation (using a piezoelectric transducer as is the case in this testing). A second sheared image is recorded with the sample in the stressed state, and the interferometric superposition of these two images creates an interferogram that represents the phase difference of neighbouring speckle sources. From this phase difference the gradient of the surface displacement can be calculated.

\section{Equipment}

An SE3 Shearography/ESPI-System produced by isi-sys $\mathrm{GmbH}$ and supplied by Limess Messtechnik und Software $\mathrm{GmbH}$ was used in this work. This system consists of a shearing head unit which contains a camera and a shearing interferometer, an LED laser diode array to provide speckle pattern illumination (using a laser wavelength of $658 \mathrm{~nm}$ ), a signal generator connected to a piezoelectric transducer used to provide vibration excitation ranging from 0 to $100 \mathrm{kHz}$ and a vacuum pump and attachment device to attach the piezoelectric transducer to test samples.

For the pulse thermography testing a TWI Thermoscope system produced by Thermal Wave Imaging Inc. using an Indigo Merlin camera was used. The Thermoscope system is an integrated pulsed thermographic system employing a medium wavelength Indigo Merlin camera described in Table 1, below. With the integrated flash system attached, the field of view of the camera is approximately $170 \times 125 \mathrm{~mm}$. 
Table 1. Camera specifications

\begin{tabular}{|l|l|}
\hline Camera name & Indigo Merlin \\
\hline Detector & Electrically cooled FPA InSb \\
\hline Array & $320 \mathrm{H} \times 256 \mathrm{~V}$ \\
\hline Spectral response & 1 to $5.4 \mu \mathrm{m}(3-5 \mu \mathrm{m}$ with cold filter $)$ \\
\hline NEdT & $<25 \mathrm{mK}(<18 \mathrm{mK}$ typically) \\
\hline Digital output & 12 -bit \\
\hline Dimensions $(\mathrm{H} \times \mathrm{W} \times \mathrm{L})$ & $140 \times 127 \times 250 \mathrm{~mm}$ \\
\hline Mass & $4.08 \mathrm{~kg}$ \\
\hline Maximum frame rate & $60 \mathrm{~Hz}$ \\
\hline
\end{tabular}

\section{Test samples}

To enable a comparison of the techniques, a set of test samples were manufactured. These samples are square flat plates of side length $200 \mathrm{~mm}$ made from aluminium, mild steel, stainless steel, CFRP and thermoplastic. Back-drilled holes of $20 \mathrm{~mm}$ diameter and varying depths were drilled in the samples to represent defects. The defect depths (i.e. material remaining after back-drilled holes were created) ranged from $0.5 \mathrm{~mm}$ to $3 \mathrm{~mm}$ in steps of $0.5 \mathrm{~mm}$. The back surface of the aluminium test sample is shown in figure 1, below. Note the large space left in the centre of the sample to allow the piezoelectric transducer to be affixed in the centre.

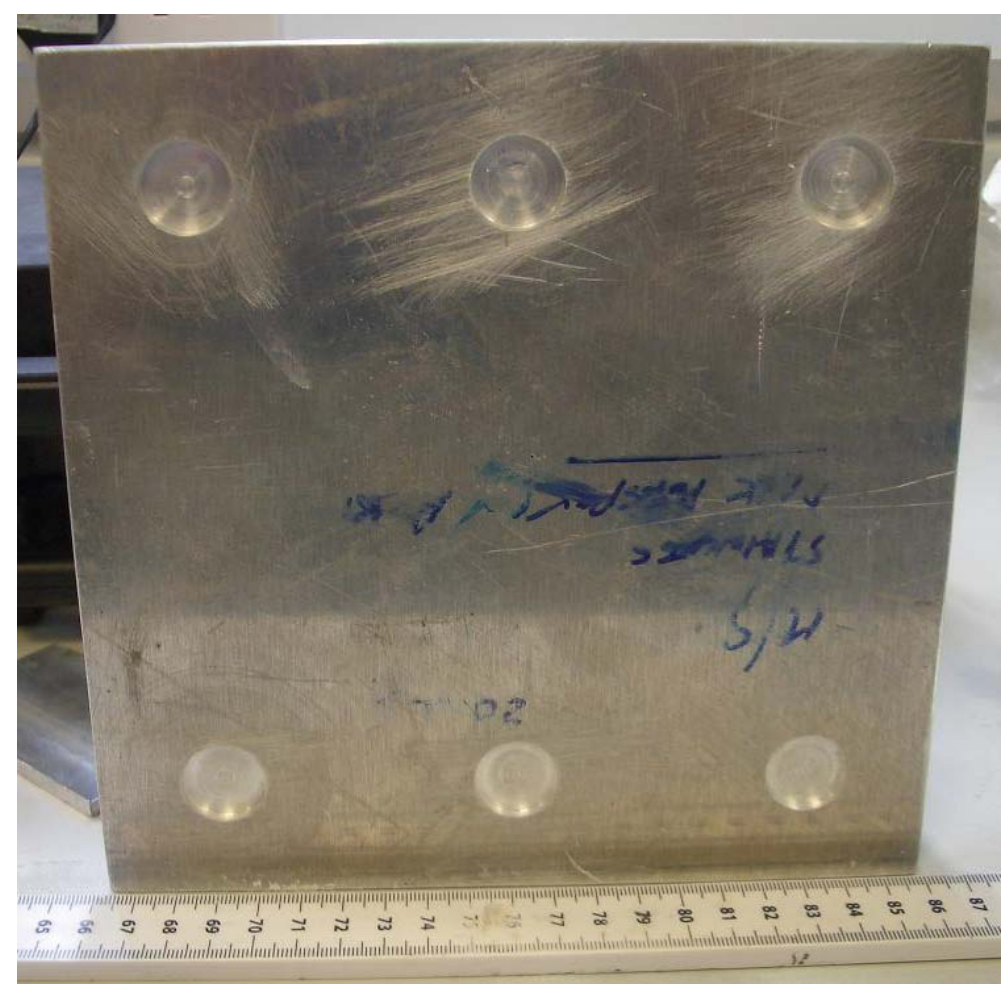

Fig 1. Aluminium test sample with $20 \mathrm{~mm} \varnothing$ back-drilled hole defects at depths from $0.5 \mathrm{~mm}$ to $3 \mathrm{~mm}$

(0.5mm to $1.5 \mathrm{~mm}$ top left to right, $2.0 \mathrm{~mm}$ to $3.0 \mathrm{~mm}$ bottom left to right)

The test sample thicknesses are shown in table 2, below.

Table 2. Sample thicknesses

\begin{tabular}{|l|c|}
\hline Test sample Material & Sample thickness \\
\hline Thermoplastic & $6.5 \mathrm{~mm}$ \\
\hline Aluminium & $6.0 \mathrm{~mm}$ \\
\hline CFRP & $4.0 \mathrm{~mm}$ \\
\hline Mild Steel & $6.5 \mathrm{~mm}$ \\
\hline Stainless Steel & $6.0 \mathrm{~mm}$ \\
\hline
\end{tabular}




\section{$4 \quad$ Flash thermography testing}

The test samples were painted with black paint to increase their emissivity/absorptivity and to reduce their reflectivity before being tested using flash thermography on their front surfaces. The thermographic data were processed and analysed using the Thermal Wave Signal Reconstruction (TWSR) method [7]. The TWSR method involves fitting a polynomial function to the logarithms of temperature change and time, and reduces temporal noise and enhances the visualisation of defects. In this case a $5^{\text {th }}$ order polynomial was used.

Figure 2 shows an example of a composite image of the thermographic data recorded for the aluminium sample. This figure shows the TWSR second derivative of temperature with respect to time. The back-drilled holes can be clearly seen as dark circular regions. The brighter regions (yellow/red) at the bottom of the image are caused by reflections from the camera and flash tube. The composite image is constructed using the data from four separate tests as the field of view of the thermographic flash system was too small to test the entire sample at once.

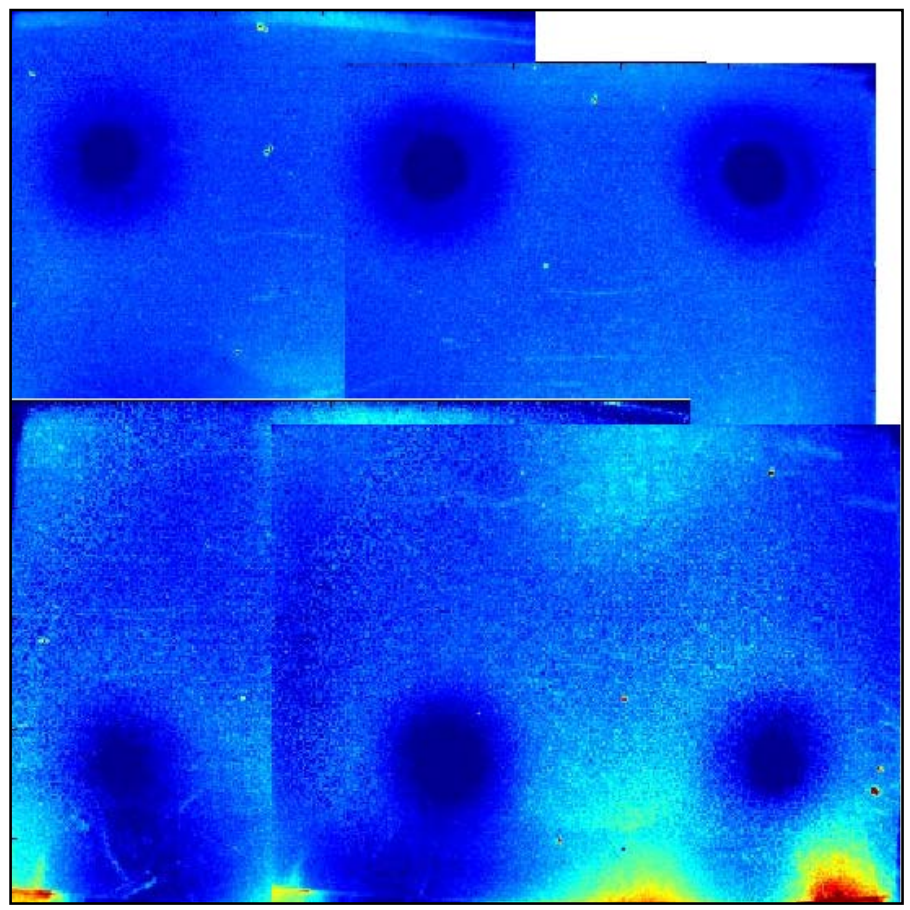

Fig 2. Composite image of flash thermography results for aluminium test sample

A signal to noise ratio analysis was performed at each defect location using raw, $1^{\text {st }}$ and $2^{\text {nd }}$ derivative TWSR data. In each case the analysis was performed as follows: A square region around each defect was selected and the temperature along each edge of the square was fitted with a $3^{\text {rd }}$ order polynomial to determine the local noise level. The noise level was calculated as twice the standard deviation of the difference between the recorded edge temperatures and the fitted polynomials. The polynomial edge equations were then used to predict the theoretical background temperature over the area containing the defect (i.e. the temperature if the defect were not present). The predicted background temperature field was subtracted from the actual temperature data to avoid any non-uniformity across the image affecting the noise and signal values. In addition, half the noise value was subtracted from the defect signal to account for the effects of noise in the defect signal itself and a $5 \times 5$ median filter was applied to remove any spatial noise. These steps were repeated for each frame (i.e. timestep) and the highest SNR result for each defect was returned.

Table 3 shows the SNR ratio results using raw thermal data.

Table 3. Flash thermography SNR results (raw data)

\begin{tabular}{|l|l|l|l|l|l|l|}
\hline Test sample Material & \multicolumn{6}{|c|}{ Defect depths (mm) } \\
\hline & \multicolumn{1}{|c|}{$\mathbf{0 . 5}$} & \multicolumn{1}{|c|}{$\mathbf{1 . 0}$} & \multicolumn{1}{c|}{$\mathbf{1 . 5}$} & $\mathbf{2 . 0}$ & $\mathbf{2 . 5}$ & $\mathbf{3 . 0}$ \\
\hline Thermoplastic & 57.20 & 27.60 & 12.34 & 12.05 & 7.57 & 4.32 \\
\hline Aluminium & 10.59 & 9.29 & 4.07 & 2.89 & 2.90 & 0.47 \\
\hline CFRP & 33.39 & 18.24 & 9.39 & 6.05 & 2.23 & 0.75 \\
\hline Mild Steel & 8.74 & 7.76 & 7.68 & 2.78 & 5.55 & 0.96 \\
\hline Stainless Steel & 11.84 & 6.66 & 6.22 & 5.52 & 2.26 & 2.08 \\
\hline
\end{tabular}


It can be seen that the SNR values decrease with increasing defect depth as expected. Using raw data contrast all defects down to depths of $2.5 \mathrm{~mm}$ can be detected in all materials (assuming a detectability threshold of SNR>1.1). It was also possible to detect $3 \mathrm{~mm}$ depth defects in the thermoplastic and stainless steel defects, but surprisingly not in the CFRP sample. The difficultly of detecting defects in the aluminium and mild steel samples was expected and is caused by their high thermal conductivities. The difficultly with CFRP was unexpected as it has a low thermal conductivity, however it can be seen from table 2 that the CFRP sample is significantly thinner than the other samples, and quite close in thickness to the deepest defect. Therefore it is theorised that the difficult here was caused by problems differentiating the defect response from that caused by the sample back wall.

Table 4 shows the SNR ratio results using $1^{\text {st }}$ derivative TWSR processed data.

Table 4. Flash thermography SNR results ( $1^{\text {st }}$ derivative)

\begin{tabular}{|l|l|l|l|l|l|l|}
\hline Test sample Material & \multicolumn{7}{|c|}{ Defect depths (mm) } \\
\hline & \multicolumn{1}{|c|}{$\mathbf{0 . 5}$} & \multicolumn{1}{c|}{$\mathbf{1 . 0}$} & \multicolumn{1}{c|}{$\mathbf{1 . 5}$} & $\mathbf{2 . 0}$ & $\mathbf{2 . 5}$ & $\mathbf{3 . 0}$ \\
\hline Thermoplastic & 93.11 & 49.67 & 26.44 & 34.81 & 15.76 & 7.00 \\
\hline Aluminium & 0.35 & 0.29 & 0.41 & 1.12 & 0.92 & 1.71 \\
\hline CFRP & 55.02 & 38.60 & 16.91 & 9.67 & 4.29 & 3.51 \\
\hline Mild Steel & 6.50 & 7.47 & 7.61 & 6.36 & 6.01 & 6.15 \\
\hline Stainless Steel & 15.00 & 13.06 & 13.19 & 11.07 & 7.86 & 5.31 \\
\hline
\end{tabular}

It can be seen that all defects other than those present in the aluminium sample have an SNR greater than 1 , and are therefore detectible. The $1^{\text {st }}$ derivative SNR values for the aluminium sample are low due to the small temperature rises generated in the sample by the flash excitation caused by its high thermal diffusivity.

Table 5 shows the SNR ratio results using $2^{\text {nd }}$ derivative TWSR processed data.

Table 5. Flash thermography SNR results $\left(2^{\text {nd }}\right.$ derivative)

\begin{tabular}{|l|l|l|l|l|l|l|}
\hline Test sample Material & \multicolumn{7}{|c|}{ Defect depths (mm) } \\
\hline & \multicolumn{1}{|c|}{$\mathbf{0 . 5}$} & \multicolumn{1}{c|}{$\mathbf{1 . 0}$} & $\mathbf{1 . 5}$ & $\mathbf{2 . 0}$ & $\mathbf{2 . 5}$ & $\mathbf{3 . 0}$ \\
\hline Thermoplastic & 20.24 & 31.52 & 19.17 & 19.38 & 11.74 & 3.37 \\
\hline Aluminium & 11.61 & 8.85 & 6.80 & 3.26 & 2.31 & 2.09 \\
\hline CFRP & 32.88 & 22.13 & 11.45 & 6.10 & 2.92 & 3.26 \\
\hline Mild Steel & 4.36 & 5.08 & 4.26 & 6.36 & 6.01 & 6.15 \\
\hline Stainless Steel & 2.74 & 2.73 & 2.48 & 3.24 & 3.17 & 2.34 \\
\hline
\end{tabular}

It should be noted that while the behaviour of the $1^{\text {st }}$ derivative SNR decreases monotonically with defect depth, the $2^{\text {nd }}$ derivative SNR is more complex and therefore the results do not decrease monotonically. However, the sensitivity of the $2^{\text {nd }}$ derivative does mean that even for the aluminium sample, which produced small temperature rises (and therefore raw data SNR), the $2^{\text {nd }}$ derivative data produces detectible SNR results for all defects and material types.

\section{$5 \quad$ Vibration excitation shearography testing}

The front surfaces of the samples were coated with white dye-penetrant developer power (i.e. chalk dust) to provide a speckle source before being tested using vibration shearography. The piezoelectric transducer was attached to the centre of the rear side of the sample and a frequency sweep was performed while observing the shearographic output image for characteristic defect indications.

Figure 3, below, shows an example composite time-averaged speckle phase image of the aluminium test sample. The image is a composite of tests performed at different excitation frequencies required to generate detectible responses from the defects located at varying depths.

The image shows time-averaged speckle phase values and has not been processed to smooth away the speckle. Data acquisition during a frequency sweep needs to be performed quite quickly considering the range of frequencies, and the time-averaged speckle phase image is what would be seen by a user of the system. 


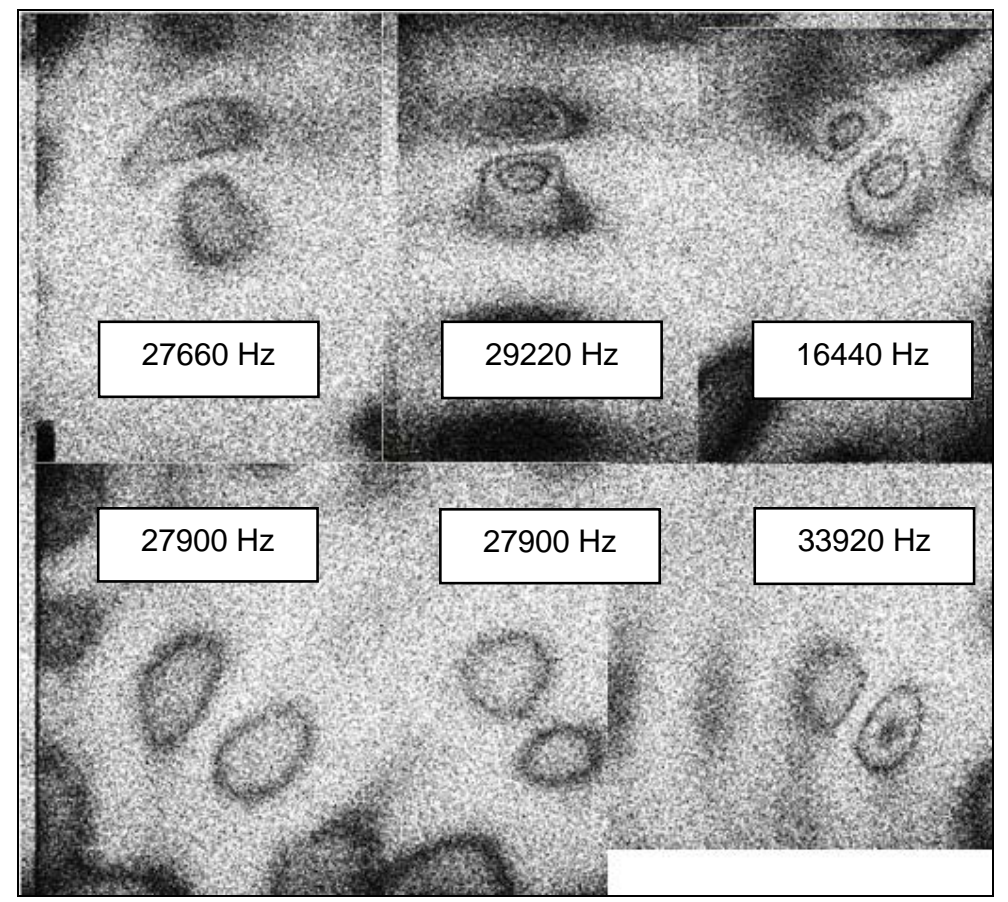

Fig 3. Composite time-averaged speckle phase image of vibration excitation shearography results

This data is known as time-averaged because the camera system is not synchronised with the excitation frequency and the camera framerate and integration times are far too slow to record data representing the state of the sample at a single point in the excitation cycle. Therefore the images seen here represent the mean absolute value of a given pixel's phase over some number of cycles. This means that the response seen in defective regions is smaller than could be recorded using an in-phase recording method, and also that the noise level over the sample is increased.

To more easily obtain a SNR, the time-average speckle phase images were smoothed to remove the speckle. This produces a continuous phase image as shown in figure 4.

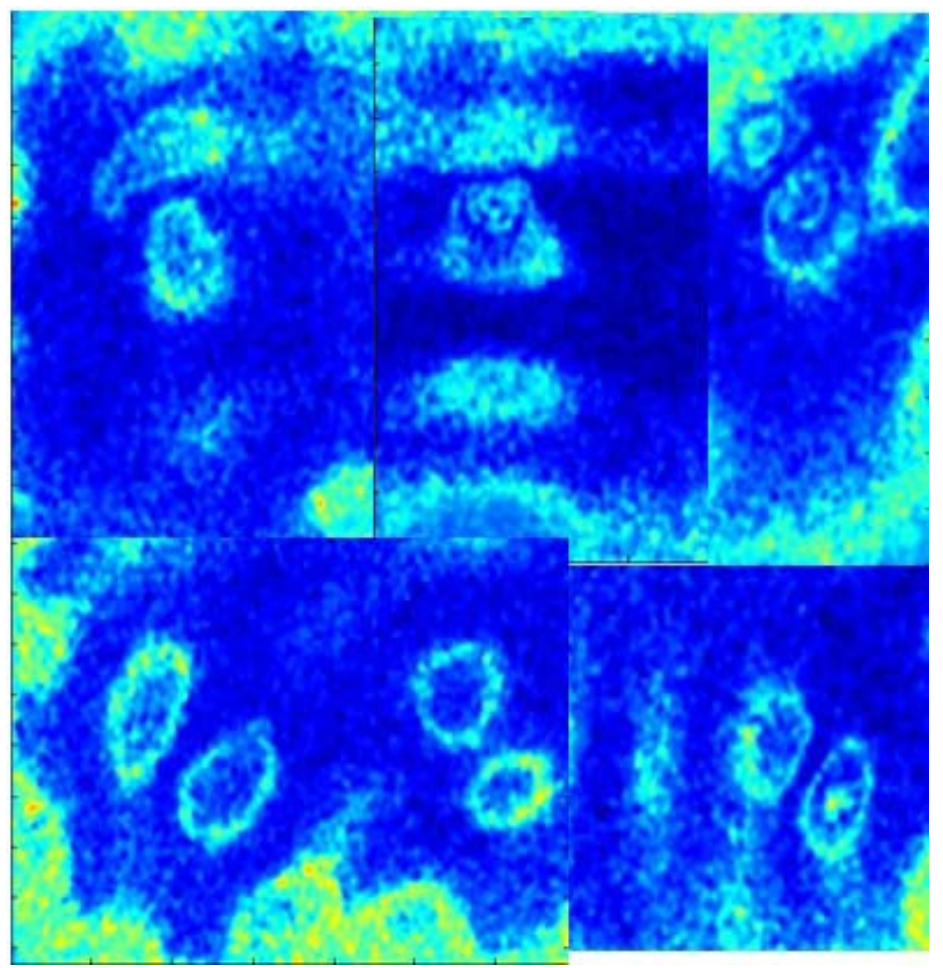

Fig 4. Smoothed composite time-averaged phase image of vibration excitation shearography results 
The shearography system used for this testing is also able to calculate instantaneous phase images (i.e. not absolute phase) using stroboscopic LED laser lighting to overcome the relatively slow camera framerate and synchronise with the excitation frequency. Unfortunately performing stroboscopic illumination takes time (approximately $10 \mathrm{~s}$ to $60 \mathrm{~s}$ per excitation frequency). Therefore performing a frequency sweep, applying stroboscopic illumination and processing for each frequency step would take too long to be a useful screening technique, and while useful when applied at the excitation frequency of a previously detected defect, this technique was not used in the current work.

A SNR analysis was carried out for the smoothed time-averaged phase shearography data, with similar processing to that carried out on the thermographic data. The differences being that variable sized rectangular regions were used to ensure that the defect mode shapes could be completely captured and that also only manually selected frames of shearographic data were processed for each defect due to software limitations.

Table 6 shows the results of the SNR analysis for the shearography data.

Table 6. Shearographic data SNR results

\begin{tabular}{|c|l|l|l|l|l|l|}
\hline Test sample Material & \multicolumn{9}{|c|}{ Defect depths (mm) } \\
\hline & $\mathbf{0 . 5}$ & $\mathbf{1 . 0}$ & $\mathbf{1 . 5}$ & $\mathbf{2 . 0}$ & $\mathbf{2 . 5}$ & $\mathbf{3 . 0}$ \\
\hline Thermoplastic & 3.85 & 2.74 & 2.88 & 5.04 & 4.04 & 3.47 \\
\hline Aluminium & 3.60 & 2.05 & 1.37 & 5.02 & 3.50 & 3.29 \\
\hline CFRP & 4.86 & 6.56 & 3.28 & - & - & - \\
\hline Mild Steel & 4.55 & 4.19 & 2.38 & 3.62 & 3.44 & 4.39 \\
\hline Stainless Steel & 4.48 & 1.95 & 2.80 & 4.36 & 3.27 & 2.47 \\
\hline
\end{tabular}

It can be seen that all of the defects could be detected except for the deepest defects in the CFRP sample. It is thought that the cause of this detection limit is the small difference in thickness between the deepest defect regions and the non-defective sample caused by the difference in sample thicknesses. In this case as the defect is close in thickness to the rest of the sample it does not appear to produce mode shapes that can be differentiated from the plate modes produced by the vibration of the whole sample.

It should also be noted that all of the SNR results for the shearographic testing are similar in magnitude and show no decreasing trend with increasing defect depth as might be expected. This is because the SNR data are generated from fringe patterns, which always have a maximum absolute value, with larger displacements simply being represented by multiple fringes. The data in question are time-averaged, which means the fringes cannot be unwrapped to generate a true phase image, therefore the maximum value detectable in these timeaveraged phase images is the value of a single fringe.

\section{$5.1 \quad$ Issues encountered}

While the frequency sweep abilities of the shearographic testing equipment allow defects of different depths and sizes (and therefore with different resonant frequencies) to be detected in what appears to be a convenient manner, there are some problems with using vibration excitation to locate defects.

In the current work, it was often found that plate modes (i.e. resonances in the entire plate rather than the region above a defect) obscured the mode shapes produced by the defects themselves. The plate modes become especially dense at higher excitation frequencies meaning that deeper and smaller defects are the ones most affected by this problem.

Figure 5 shows a time-averaged speckle phase image of part of the aluminium sample. In this image a defect can be seen in the top right corner of the image. However, it is both difficult to see this defect and also to differentiate between the high density of plate modes seen in this image and any other possible defect signals. 


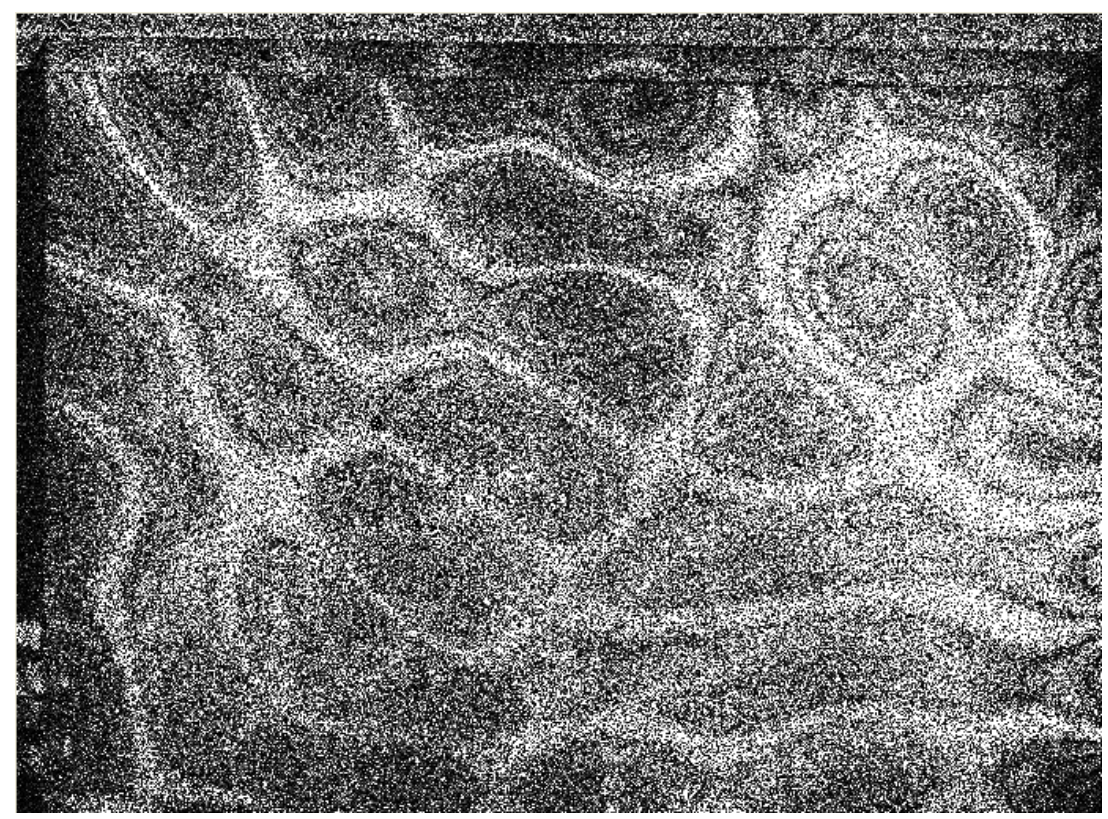

Fig 5. Time averaged phase image of defect in aluminium sample $(27600 \mathrm{~Hz})$

\section{Conclusions}

Flash thermographic testing and vibration excitation shearographic testing was carried out on flat plate samples made of aluminium, mild steel, stainless steel, CFRP and thermoplastic containing back-drilled flatbottomed hole artificial defects.

Flash thermography using unprocessed thermal contrast data was able to detect all of the defects in all of the sample materials except for the $3.0 \mathrm{~mm}$ defects in the CFRP, mild steel and aluminium samples. It is thought that the difficulties in detecting the $3.0 \mathrm{~mm}$ defect in CFRP were caused by interference of the back wall signal due to the shallow nature of the back-drilled holes used to create these defects, and in the case of the mild steel and aluminium samples due to their high thermal diffusivities reducing the signal level. Using $2^{\text {nd }}$ derivative TWSR processing on the thermal data it was possible to detect all of the defects.

The vibration excitation shearography data was recorded and processed in its time-average phase form. This necessarily reduces the signal level and increases noise, however performing stroboscopic illumination and phase stepping at every increment in a frequency sweep would take far too long to be practicable. Using this shearography data it was not possible to detect defects deeper than $1.5 \mathrm{~mm}$ in the CFRP sample, but it was possible to detect all of the other defects. It is thought this detection limit was also caused by the very shallow nature of the back-drilled holes used to create these defects. The CFRP sample was significantly thinner than the other samples (see table 2), and therefore these defects were closer to the thickness of the sample. This is thought to have caused interference with both the thermal response and the vibration response of these defects. This effect may impact on the applicability of both flash thermography and vibration excitation shearography for wall thickness-type measurements. Further work is currently being carried out to determine the depth/diameter limits of detectability for both techniques using smaller diameter and deeper back-drilled hole defects.

In the current work the noise level of the shearographic results was measured in the region surrounding the locations of the defects. This is not applicable to the testing of unknown defects and work is proceeding to use the shearographic data returned at different vibration frequencies to automatically locate defects.

During the vibration excitation shearographic testing it was found that plate modes made detecting the vibration responses caused by defects difficult. The density of plate modes increases with excitation frequency and higher excitation frequencies are required to generate resonances in deeper/smaller diameter defects, therefore it is possible this will produce a fundamental limit to the size and depth of defects that can be detected using this technique. The presence of these plate modes may limit the effectiveness of shearography as an NDE tool for certain combinations of sample shape, material type and defect size and depth.

It should however, be noted that the author has found that real-world structures containing actual BVID damage, which are often curved (e.g. aircraft wing outer structures), produce both fewer plate modes and wider ranging defect resonances making these easier to detect. Further work will be carried out to investigate these phenomena. 


\section{$7 \quad$ Acknowledgments}

This work reported here is a part of the core research programme of the UK Research Centre in NonDestructive Evaluation (RCNDE) funded by the Engineering and Physical Science Research Council (EPSRC). The authors also acknowledge EPSRC for an equipment grant used to purchase the shearography system used in this work.

\section{References}

[1] Milne JM, Reynolds WN. The non-destructive evaluation of composites and other materials by thermal pulse video thermography. Proc. Soc. Photo-Opt. Instrum. Eng, 1985. pp.119-122.

[2] Lau SK, Almond DP, Milne JM. A quantitative analysis of pulsed video thermography. Non Dest. Testing Eval. Int. 24, 1991, pp.195-202.

[3] Shepard S. Advances in Pulsed Thermography. A.E. Rozlosnik, R.B. Dinwiddie eds, Thermosense XIV, SPIE Proc. 4360, 2001: pp.511-515.

[4] Steinchen, W., Yang, LX. (2003). Digital Shearography: Theory and Application of Digital Speckle Pattern Shearing Interferometry, SPIE Press, Bellingham, Washington USA.

[5] Hung, YY., Chen, YS., Ng, SP., Liu, L., Huang, YH., Luk, BL., Ip, RWL, Wu, CML, Chung, PS. (2009). Review and comparison of shearography and active thermography for nondestructive evaluation. Materials Science and Engineering R 64, pp.73-112. doi:10.1016/j.mser.2008.11.001

[6] Gryzagoridis, J., Findeis, D. (2007). Benchmarking shearographic NDT for composites. Insight Vol 50 No 5 May 2008, pp.249-252. DOI: 10.1784/insi.2008.50.5.249

[7] Steven M. Shepard, James R. Lhota, Bruce A. Rubadeux, David Wang, and Tasdiq Ahmed, Reconstruction and enhancement of active thermographic image sequences, Opt. Eng., Vol. 42, 1337 (2003); DOI:10.1117/1.1566969 